\title{
Baseline tumor Lipiodol uptake after transarterial chemoembolization for hepatocellular carcinoma: identification of a threshold value predicting tumor recurrence
}

\author{
Yusuke Matsui ${ }^{1,2}$, Masahiro Horikawa ${ }^{1}$, Younes Jahangiri Noudeh¹, John A. Kaufman ${ }^{1}$, \\ Kenneth J. Kolbeck ${ }^{1}$, Khashayar Farsad ${ }^{1}$ \\ ${ }^{1}$ Dotter Interventional Institute, Oregon Health and Science University, 3181 SW Sam Jackson Park Rd, Portland, USA \\ 2 Department of Radiology, Okayama University Medical School, 2-5-1 Sikata-cho, Kita-ku, Okayama 700-8558, Japan
}

Radiol Oncol 2017; 51(4): 393-400.

Received 16 April 2017

Accepted 30 June 2017

Correspondence to: Yusuke Matsui, Department of Radiology, Okayama University Medical School, 2-5-1 Sikata-cho, Kita-ku, Okayama 700-8558, Japan. Phone: +81 86235 7313; Fax: +81 86235 7316; E-mail: y-matsui@okayama-u.ac.jp

Disclosure: Financial activities related to the present article: Khashayar Farsad received a grant from Guerbet for this study. Financial activities not related to the present article: Yusuke Matsui reports a grant from Japan Radiological Society supported by Bayer Yakuhin, a consultancy fee from Stryker Japan, a lecturer's fee from Terumo Corporation; John A. Kaufman reports grants from NIH, WL Gore, and BTG, personal fees from VIVA Physicians, Delcath, MarrowStim and COOK Medical, book royalties and journal editor stipend from Elsevier, section editor stipend from ARRS/AJR, stock ownership in Hatch Medical, Vu Medi, Endoshape, and Veniti, stock options in Javelin Medical, AV Medical, and BIO2 Medical; Kenneth J. Kolbeck reports a grant from Guerbet. A summary of this work was presented at the Society of Interventional Radiology (SIR) 2016 annual meeting (http://dx.doi.org/10.1016/j.jvir.2015.12.227).

Background. The aim of the study was to evaluate the association between baseline Lipiodol uptake in hepatocelIular carcinoma (HCC) after transarterial chemoembolization (TACE) with early tumor recurrence, and to identify a threshold baseline uptake value predicting tumor response.

Patients and methods. A single-institution retrospective database of HCC treated with Lipiodol-TACE was reviewed. Forty-six tumors in 30 patients treated with a Lipiodol-chemotherapy emulsion and no additional particle embolization were included. Baseline Lipiodol uptake was measured as the mean Hounsfield units (HU) on a CT within one week after TACE. Washout rate was calculated dividing the difference in HU between the baseline CT and follow-Up CT by time (HU/month). Cox proportional hazard models were used to correlate baseline Lipiodol uptake and other variables with tumor response. A receiver operating characteristic (ROC) curve was used to identify the optimal threshold for baseline Lipiodol uptake predicting tumor response.

Results. During the follow-up period (mean 5.6 months), 19 (41.3\%) tumors recurred (mean time to recurrence $=3.6$ months). In a multivariate model, low baseline Lipiodol uptake and higher washout rate were significant predictors of early tumor recurrence $(P=0.001$ and $<0.0001$, respectively $)$. On ROC analysis, a threshold Lipiodol uptake of 270.2 HU was significantly associated with tumor response (95\% sensitivity, $93 \%$ specificity).

Conclusions. Baseline Lipiodol uptake and washout rate on follow-up were independent predictors of early tumor recurrence. A threshold value of baseline Lipiodol uptake > $270.2 \mathrm{HU}$ was highly sensitive and specific for tumor response. These findings may prove useful for determining subsequent treatment strategies after Lipiodol TACE.

Key words: transarterial chemoembolization; hepatocellular carcinoma; Lipiodol, tumor response, threshold

\section{Introduction}

Transarterial chemoembolization (TACE) has been proven effective for treatment of intermediate stage hepatocellular carcinoma (HCC). ${ }^{1,2}$ Lipiodol, a di- iodinated ethyl ester derivative of poppy seed oil, has been widely used in the standard TACE procedure. ${ }^{3}$ When administered selectively via the hepatic artery, Lipiodol accumulates preferentially in HCC and is retained for a longer period than in the 
background liver parenchyma. ${ }^{4-6}$ Because Lipiodol demonstrates trans-sinusoidal penetration, arterial injection of Lipiodol can temporarily achieve dual arterial and portal embolization, unlike other particulate embolic materials such as microspheres. ${ }^{3,7}$ An association between intratumoral Lipiodol accumulation, pathological tumor necrosis ${ }^{8-12}$ and local recurrence rate ${ }^{13-15}$ has been shown in retrospective analyses; however, no study has investigated a specific value of baseline Lipiodol uptake, i.e., the Lipiodol uptake immediately after TACE, predicting tumor recurrence. If a specific baseline Lipiodol uptake value proves useful to predict tumor recurrence, it should have significant meaning for subsequent clinical management of HCC patients. This study examined whether baseline Lipiodol uptake in HCC was associated with tumor response, and whether a cutoff value of Lipiodol uptake could be identified predicting tumor recurrence.

\section{Patients and methods}

\section{Study population}

Retrospective single institution database review was performed of patients with primary HCC who underwent Lipiodol-TACE from April 2007 to August 2013. Institutional review board approval was obtained. Inclusion criteria were i) documented non-contrast computed tomography (CT) of the liver within one week after TACE, ii) at least one documented triphasic contrast CT including noncontrast, arterial and delayed phase imaging on follow-up for evaluation of tumor recurrence, iii) lesion diameter $\geq 10 \mathrm{~mm}$, and iv) lesions stained entirely and homogeneously with Lipiodol after TACE. Exclusion criteria were i) patients with more than 6 lesions, ii) lesions stained partially or heterogeneously with Lipiodol; those lesions were not suitable for the Lipiodol uptake measurement because of the nonuniform Lipiodol concentration and possibility of incomplete treatment, iii) lesions treated with additional particulate embolic materials, iv) lesions for which a second TACE or any additional treatment including surgery, radiofrequency ablation (RFA), radiation therapy, or systemic therapy (e.g., sorafenib) was performed before the initiation of follow up. Forty-six tumors in 30 patients meeting criteria were evaluated.

\section{TACE procedure}

Selective catheterization of lobar, segmental or subsegmental hepatic arteries supplying tumor was performed under standard fluoroscopic guidance using 5-6 Fr base catheters in the first order aortic branch (celiac or superior mesenteric artery) followed by microcatheter selection of higher order branches as appropriate. A Lipiodol-chemotherapy emulsion (doxorubicin or epirubicin, 50mg) was prepared by admixture in a 1:1 or 2:1 ratio using a three-way stopcock and injected into selected hepatic arteries through the microcatheter. The endpoint of injection was stasis of blood flow in the feeding artery or visualization of trans-sinusoidal portal branches. The volume of Lipiodol injected ranged from 1 to $10 \mathrm{~mL}$ per lesion. Lipiodol was used as the sole embolic material; no additional embolization with other particulate embolic materials was performed. Although this method corresponds to "chemo-lipiodolization" in European guidelines ${ }^{16}$ or "Lip-TAI (transcatheter arterial infusion)" in Japanese guidelines ${ }^{17}$, "TACE" was employed as a unified term in this study. The embolic particles were not routinely used in our institutional practice because our institutional data (not shown) demonstrated patients' survival outcome was not significantly different using particulate embolization.

\section{Data collection and image evaluation}

Patient data including age, sex, etiology of liver disease, Child-Pugh classification, and Barcelona Clinic Liver Cancer (BCLC) staging were collected from the electronic medical record. Procedural details recorded included selectivity of treatment (lobar vs. subsegmental/segmental), volume of Lipiodol delivered and amount of chemotherapeutic delivered. Image evaluation was performed using a Digital Imaging and Communication in Medicine (DICOM) viewer (IMPAX; Agfa, Mortsel, Belgium). Lipiodol uptake was measured using the Hounsfield units (HU) of the target lesion on a noncontrast CT taken within one week after TACE and on every follow-up CT. The HUs on the CT within one week after TACE represented the baseline Lipiodol uptake. The follow-up interval varied case by case. The HUs were measured by hand drawing a region of interest (ROI) along the lesion periphery at the axial slice with the tumor maximum diameter, using the contrast-enhanced pre-procedure diagnostic scan as a reference. ROI analysis was performed with consistent contrast window level (WL) and width (WW) (WL/WW = 50/350). The diameter of the ROI on the baseline CT was used as the lesion size, since only lesions demonstrating entire and homogeneous uptake were included in the analysis. Lipiodol washout was defined as de- 
creased density of Lipiodol in a part of the tumor or uniformly within the entire tumor, and evaluated using HU analysis on every available follow-up CT. The degree of washout was quantitatively evaluated by calculating the HU difference between the baseline $\mathrm{CT}$ and the follow-up $\mathrm{CT}$ at which washout was initially detected. If the HUs within the tumor on the last follow-up CT were greater than at baseline $\mathrm{CT}$, as was occasionally seen with contracting tumors after treatment, the quantitative washout was recorded as $1.0 \mathrm{HU}$. The washout rate was calculated by dividing the quantitative washout by the follow up time period (HU/month). Tumor recurrence was defined as the appearance of new arterial enhancement within or along the margins of a completely treated lesion on the follow-up triphasic contrast CT. Evaluation was terminated when additional treatment was performed or when recurrence was identified. Two board-certified radiologists independently performed all image evaluation, with discrepancies decided by consensus with a third radiologist. Mean values of two measurements were used in all analyses.

\section{Statistical analysis}

Numerical data were summarized as mean \pm standard deviation (SD) and the categorical variables were shown as frequency (percentage). Interobserver reliability of measurements was assessed by Cronbach's alpha ${ }^{18}$, and standard error of the measurement was calculated using the formula: $S E M=S D \times \sqrt{1-r_{x x}}$, where SEM is standard error of measurement, SD is standard deviation and rxx demonstrates the reliability of the test represented by Cronbach's alpha ${ }^{19}$. Analyses for predictors of tumor response and estimation of the cutoff lipiodol uptake value predicting tumor response were performed on lesion-by-lesion basis. Uni- and multivariate Cox proportional hazard models were created to evaluate the predictability of baseline Lipiodol uptake for tumor response. In addition to the baseline Lipiodol uptake, other variables such as lesion size, treatment selectivity and washout rate were subjected to the univariate model. Variables with a $P$ value $<0.200$ were entered in the multivariable model. Independent samples t-test was used to compare the mean baseline Lipiodol uptake between the tumors with and without recurrence, as well as between the degrees of treatment selectivity. The median recurrencefree interval was defined, and tumor recurrence before the median recurrence-free interval was defined as early recurrence. Baseline Lipiodol up-
TABLE 1. Summary of the study population

\begin{tabular}{|c|c|c|}
\hline Characteristics & & \\
\hline \multicolumn{3}{|l|}{ Patients } \\
\hline Age (years) & & $59.9 \pm 7.9$ \\
\hline \multirow{2}{*}{ Gender } & Female & $12(40.0 \%)$ \\
\hline & Male & $18(60.0 \%)$ \\
\hline \multirow{5}{*}{ Liver disease etiology } & $\mathrm{HCV}$ & $13(43.3 \%)$ \\
\hline & $\mathrm{HCV}+\mathrm{Alcohol}$ & 7 (23.3\%) \\
\hline & Alcohol & $4(13.3 \%)$ \\
\hline & HBV & $1(3.3 \%)$ \\
\hline & Other & $5(16.7 \%)$ \\
\hline \multirow{2}{*}{ Child-Pugh class } & A & $10(33.3 \%)$ \\
\hline & B & $20(66.7 \%)$ \\
\hline \multirow{2}{*}{ BCLC stage } & A & $17(56.7 \%)$ \\
\hline & B & $13(43.3 \%)$ \\
\hline \multicolumn{3}{|l|}{ Tumors } \\
\hline Baseline tumor size (mm) & & $20.3 \pm 10.1$ \\
\hline \multirow{3}{*}{ Treatment selectivity } & Lobar & $16(34.8 \%)$ \\
\hline & Segmental & $22(47.8 \%)$ \\
\hline & Sub-segmental & $8(17.4 \%)$ \\
\hline Baseline Lipiodol uptake (HU) & & $328.3 \pm 185.2$ \\
\hline Lipiodol uptake at final evaluation (HU) & & $205.0 \pm 219.5$ \\
\hline Washout (HU) & & $136.9 \pm 127.6$ \\
\hline Follow-up time (months) & & $5.6 \pm 6.2$ \\
\hline \multirow{2}{*}{ Tumor recurrence } & Yes & $19(41.3 \%)$ \\
\hline & No & 27 (58.7\%) \\
\hline
\end{tabular}

The numerical data were summarized as mean \pm standard deviation and the categorical data were shown as frequency (percentage). $\mathrm{BCLC}=$ Barcelona clinic liver cancer; $\mathrm{HBV}=$ hepatitis $B$ virus; $\mathrm{HCV}=$ hepatitis $\mathrm{C}$ virus; $\mathrm{HU}=$ Hounsfield unit

take and washout rates were compared between tumors with and without early recurrence using the independent samples t-test. Receiver operating characteristic (ROC) curve analysis was used to determine the optimal cutoff point for baseline Lipiodol uptake predicting tumor recurrence, and for Lipiodol washout rates predicting early tumor recurrence. Natural logarithm values of the numerical exposure variables were used in Cox regression models, and $P$ values $<0.05$ were considered statistically significant. All statistical analyses were done using Stata IC version 13.1 for Windows.

\section{Results}

The study population is summarized in Table 1 . The mean follow-up period was 5.6 months (range, 
TABLE 2. Results of cox regression analysis to predict factors for tumor recurrence

\begin{tabular}{|c|c|c|c|c|}
\hline \multirow{2}{*}{ Predicting factor } & \multicolumn{2}{|c|}{ Univariate model } & \multicolumn{2}{|c|}{ Multivariate model" } \\
\hline & Hazard ratio $(\mathrm{Cl})$ & $p$ Value & Hazard ratio $(\mathrm{Cl})$ & $p$ Value \\
\hline Lesion size (mm) & $0.70(0.24-2.09)$ & 0.527 & - & - \\
\hline Baseline Lipiodol uptake (HU) & $0.37(0.19-0.70)$ & 0.002 & $0.002(0.0-0.087)$ & 0.001 \\
\hline Presence of Lipiodol washout & $N A^{* *}$ & NA & - & - \\
\hline
\end{tabular}

* Variables with a p value less than 0.200 were entered in the multivariate model. ** Not calculated because no tumors without Lipiodol washout recurred while $65.5 \%$ ( $19 / 29$ ) of the tumors with Lipiodol washout recurred. $\mathrm{Cl}=$ confidence interval; $\mathrm{HU}=$ Hounsfield unit

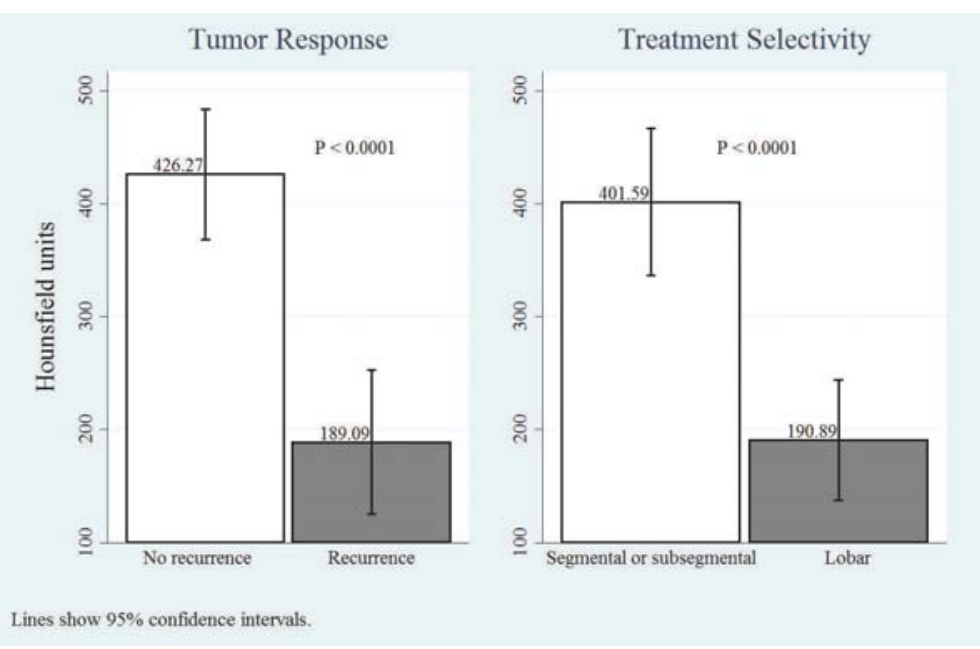

FIGURE 1. Baseline Lipiodol uptake after TACE for HCC. Significantly higher Lipiodol uptake was noted in tumors without recurrence and in tumors treated selectively with TACE.

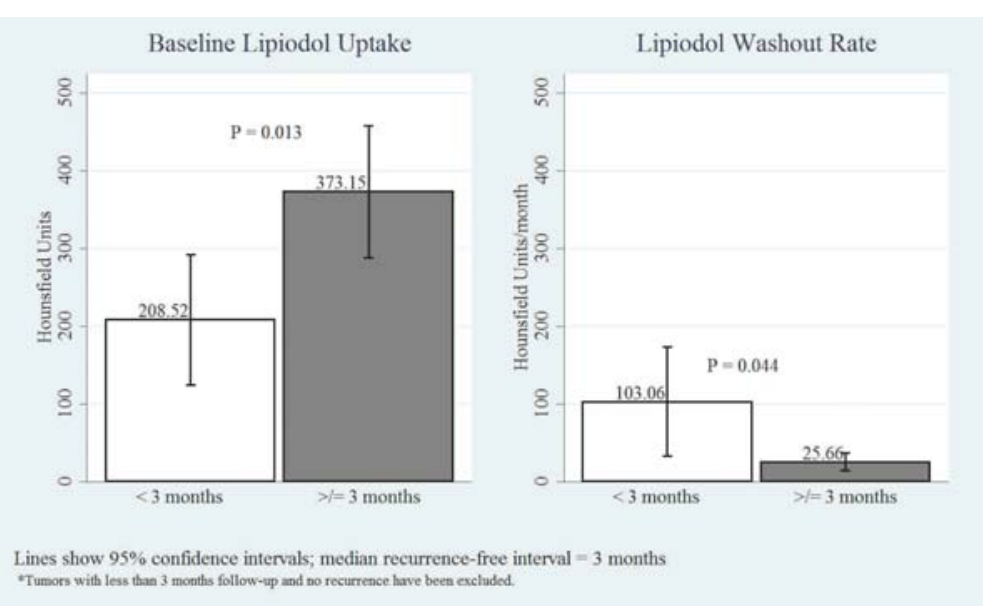

FIGURE 2. Baseline Lipiodol uptake and washout rate in relation to early tumor recurrence. Early recurring tumors demonstrated significantly lower baseline Lipiodol uptake and higher Lipiodol washout rates relative to tumors recurring after the median recurrence free interval (= 3 months) and tumors with no recurrence.
1.1-37.6). Nineteen (41.3\%) lesions recurred, with a mean time to recurrence of 3.6 months (range, 1.115.1) and a median recurrence-free interval of 3.0 months. The mean baseline Lipiodol uptake was $328.3 \pm 185.2 \mathrm{HU}$. The mean quantitative washout of Lipiodol during the entire follow-up period was $136.9 \pm 127.6 \mathrm{HU}(58.4 \pm 89.5 \mathrm{HU}$ per month). Interobserver reliability was high amongst the two independent radiologists for measuring Lipiodol uptake (Cronbach's alpha $=0.994)$, with a standard error of measurement of $14.3 \mathrm{HU}$.

\section{Predictors of tumor response}

No recurrences were seen in tumors with uniformly retained Lipiodol at follow up imaging. Of the 29 tumors with visible washout on follow-up imaging, 19 (65.5\%) demonstrated recurrence. In univariate analysis, lower baseline Lipiodol uptake and greater Lipiodol washout rate were statistically significant predictors of earlier time to tumor recurrence (Table 2, $P=0.002$ and $<0.0001$, respectively). Performing a non-selective (lobar) TACE was also significantly associated with earlier time to recurrence compared to selective (segmental/ subsegmental) TACE (Table 2, $P=0.001$ ). In multivariate Cox regression analysis, lower baseline Lipiodol uptake and greater Lipiodol washout rate were both significant predictors of earlier time to tumor recurrence (Table 2, $P=0.001$ and $<0.0001$, respectively).

The mean baseline Lipiodol uptake of tumors without recurrence was significantly higher than in tumors demonstrating recurrence (Figure 1; $426.3 \pm$ $148.8 \mathrm{HU}$ vs. $189.1 \pm 137.5 \mathrm{HU}, P<0.0001)$. Tumors treated with selective TACE demonstrated significantly higher baseline Lipiodol uptake compared with tumors treated with lobar TACE (Figure 1; $401.6 \pm 32.4 \mathrm{HU}$ vs. $190.9 \pm 26.4 \mathrm{HU}, P<0.0001$ ). Baseline Lipiodol uptake was significantly lower in 
tumors recurring before the median recurrence-free interval (e.g., early tumor recurrence) compared to tumors recurring after the median recurrence-free interval and tumors without recurrence (Figure 2; $208.5 \pm 154.8$ HU vs. $373.2 \pm 200.3 \mathrm{HU}, P=0.013$ ). By comparison, the rate of Lipiodol washout at follow up was significantly greater in early tumor recurrence compared to tumors recurring after the median recurrence-free interval and tumors without recurrence (Figure 2; $103.1 \pm 129.1 \mathrm{HU} /$ month vs. $25.7 \pm 26.6 \mathrm{HU} /$ month, $\mathrm{P}=0.044$ ).

\section{Estimating the cutoff Lipiodol uptake value predicting tumor recurrence}

Using the area under the ROC curve for post-TACE baseline Lipiodol uptake, an estimated threshold Lipiodol uptake value of $270.2 \mathrm{HU}$ was identified below which there was a high probability of tumor recurrence and above which there was a high probability of tumor response over the mean follow-up time of 5.6 months with a sensitivity of $95 \%$ and specificity of $93 \%$ (Figure 3). Comparison of the cumulative hazards of tumor recurrence over the follow-up period also showed significantly higher hazards of recurrence in tumors with Lipiodol uptake $<270.2 \mathrm{HU}$ after TACE (Figure 4; $\mathrm{P}<0.0001$ ). ROC curve analysis for follow up Lipiodol washout rate revealed a value of $37.8 \mathrm{HU}$ per month above which there was a high probability of early tumor recurrence with a sensitivity of $78 \%$ and specificity of $74 \%$ (Figure 5). The cumulative hazards for tumor recurrence were significantly higher in tumors with a washout rate $>37.8 \mathrm{HU}$ per month (Figure 6; $\mathrm{P}=0.001$ ).

\section{Discussion}

In this study, we found a strong correlation between baseline Lipiodol uptake after TACE for HCC and tumor response. The baseline uptake was that defined by the density of Lipiodol tumor stain on non-contrast CT imaging within the first week after TACE. The critical threshold value for baseline Lipiodol uptake predicting tumor recurrence vs. response in this cohort was $270.2 \mathrm{HU}$ with a high degree of sensitivity and specificity (95\% and $93 \%$, respectively) over the roughly 6 months mean follow up interval. Post-TACE Lipiodol uptake has been shown to predict necrosis in HCC lesions..$^{8-12}$ Moreover, low or incomplete Lipiodol uptake has been shown to predict tumor recurrence. ${ }^{13-15}$ The present study contributes to this body of knowl-

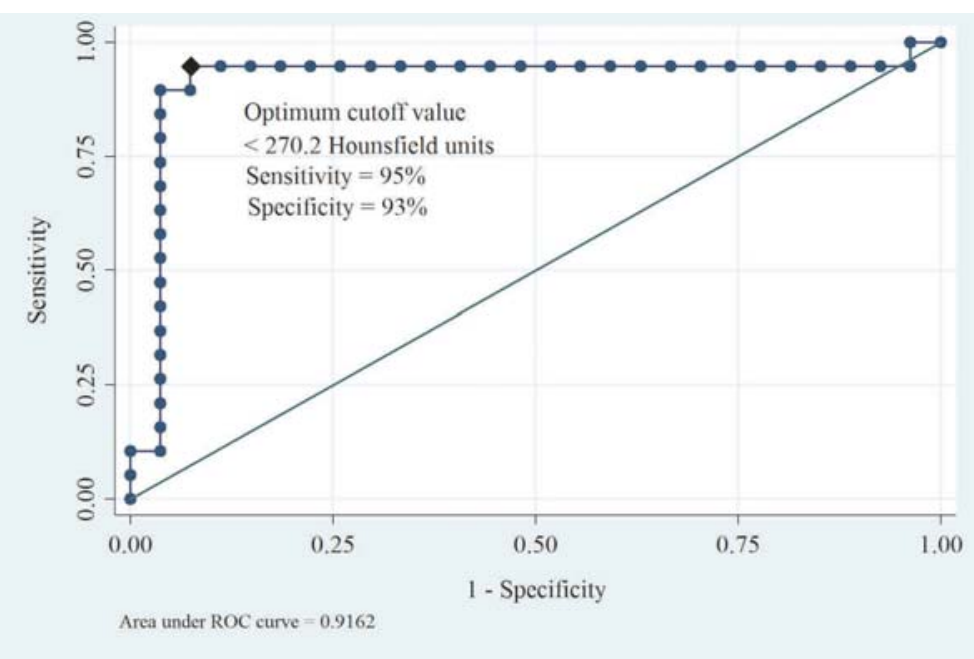

FIGURE 3. ROC curve showing the area under curve and optimal cutoff value of baseline Lipiodol uptake after TACE to predict tumor recurrence.

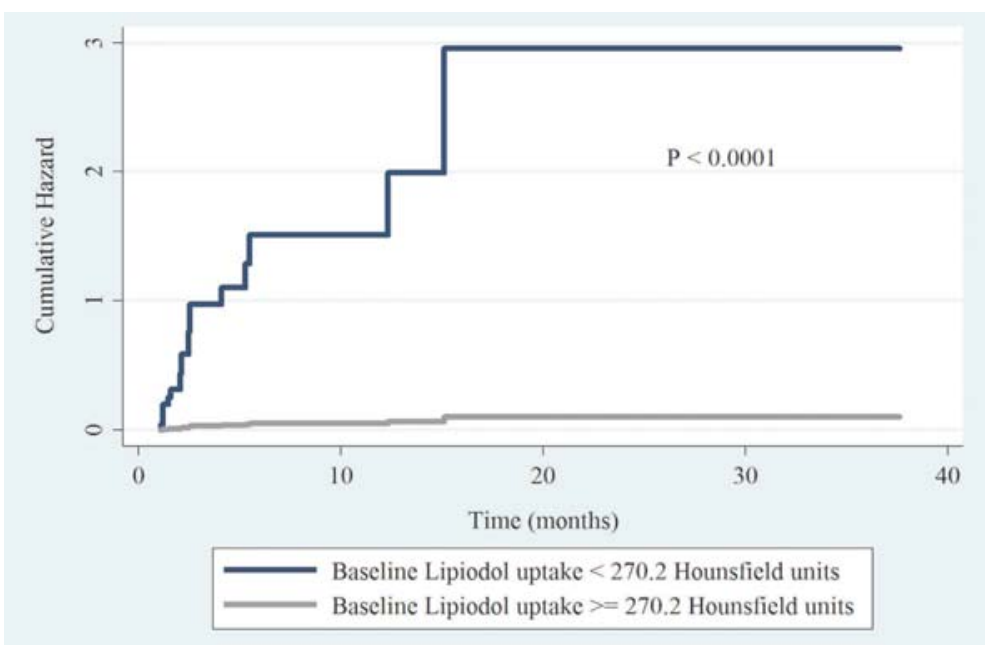

FIGURE 4. Comparison of the cumulative hazards of tumor recurrence over the follow-up period between baseline Lipiodol uptake above and below the identified threshold. Tumors with Lipiodol uptake $<270.2 \mathrm{HU}$ demonstrated significantly higher hazards of recurrence after TACE.

edge by focusing on the baseline uptake at the time of TACE, and identifies a threshold value of 270.2 HU on baseline post-TACE non-contrast CT imaging as a predictor of tumor recurrence. Moreover, our cohort involved TACE using Lipiodol as the sole embolic agent, therefore demonstrating the effect of Lipiodol accumulation independently from additional embolic agent effects.

We found only one previous study discussing a critical cutoff point of post-TACE Lipiodol uptake 


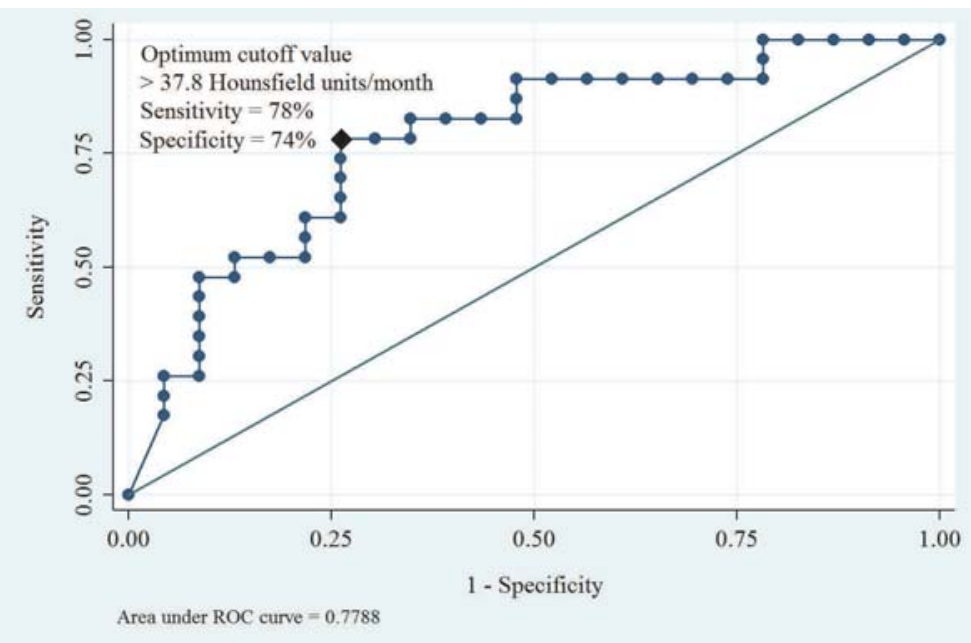

FIGURE 5. ROC curve showing the area under curve and optimal cutoff value of Lipiodol washout rate after TACE to predict early tumor recurrence.

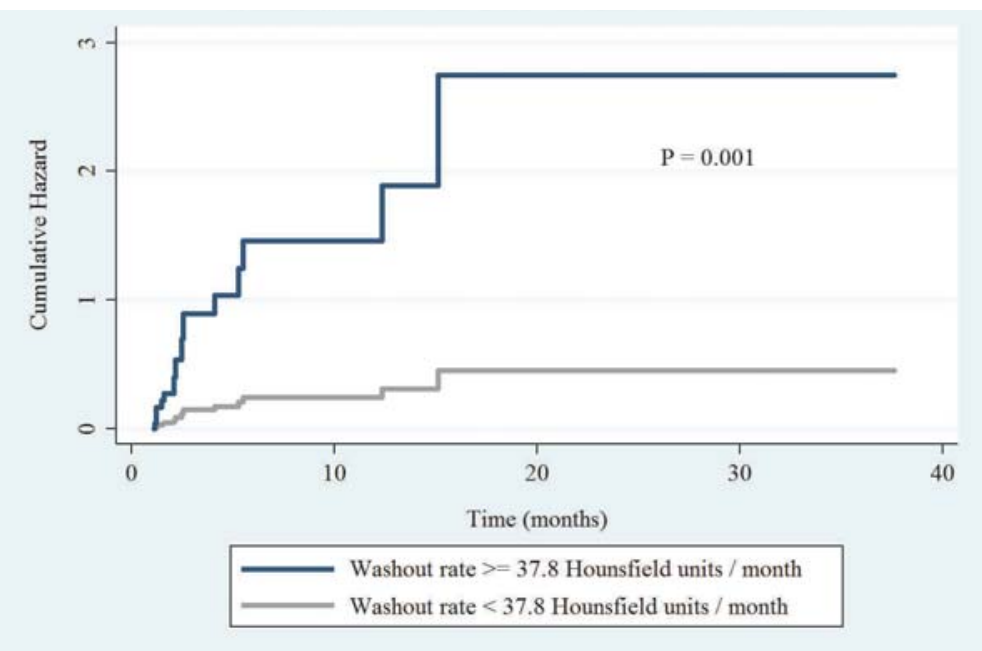

FIGURE 6. Comparison of the cumulative hazards of tumor recurrence over the follow-up period between Lipiodol washout rates above and below the identified threshold. Tumors with Lipiodol washout rates $\geq 37.8 \mathrm{HU} / \mathrm{month}$ demonstrated significantly higher hazards of recurrence after TACE.

associated with tumor necrosis..$^{12}$ Imaeda et al. ${ }^{12}$ evaluated the histological necrosis level in 12 autopsies and 23 surgical resection specimens from patients with HCC who had undergone TACE. They reported that uniform dense retention of Lipiodol was observed on CT 3-4 weeks after TACE in 12 out of 15 cases with massive ( $\geq 97 \%$ ) histological necrosis and in only one out of 20 cases with nonmassive necrosis $(P<0.01)$, showing a Lipiodol uptake value of $365 \mathrm{HU}$ as a cutoff value for massive necrosis with $89 \%$ sensitivity and $73 \%$ specificity. ${ }^{12}$
The cutoff value identified in our study is less than that found in this study, which may be related to the differences in TACE technique, study methods and outcomes. For example, we used the baseline Lipiodol uptake (within one week of treatment) as the input variable, and tumor recurrence by $\mathrm{CT}$ as the main outcome variable. Furthermore, we used follow-up data from larger number of lesions (46 tumors in 30 patients). We have observed that lesion contraction over time can create a greater density of Lipiodol stain compared to baseline values in tumors responding to therapy, which may partially explain the higher threshold value observed by Imaeda et al. when evaluating scans obtained approximately one month after TACE.

We found the Lipiodol washout rate, defined as loss of HU per month, was also a strong and independent predictor of early tumor recurrence. A similar finding has been observed in another study after evaluation of 42 HCC patients who were scheduled for liver transplantation. ${ }^{20}$ The authors subjectively classified Lipiodol washout on CT into complete, strong, moderate, and poor, and found that a moderate or strong Lipiodol washout was correlated with lower degrees $(\leq 75 \%)$ of tumor necrosis. ${ }^{20}$ In the present study, we identified a washout rate of $37.8 \mathrm{HU}$ above which there was a high risk of early tumor recurrence over the follow up period in our cohort.

Lipiodol can pass through arterio-portal communications such as the peribiliary plexus, terminal arterial-sinusoidal branches, portal vaso vasori, and direct arterioportal anastomoses.,7 Consequently, transarterial delivery of Lipiodol results in embolization of feeding vessels from both the arterial and portal sides of the tumor. ${ }^{3,7}$ Another role of Lipiodol is that of a carrier of the chemotherapeutic agent. ${ }^{7}$ In this regard, higher Lipiodol uptake may represent enhanced embolization of tumor blood supply and also contribute to a higher local concentration of chemotherapeutic agents in the tumor.

Cone-beam CT (CBCT) has been shown to demonstrate tumor Lipiodol accumulation with TACE, and HUs measured on CBCT have been shown to be comparable to multidetector CT. ${ }^{21}$ The use of intraprocedural CBCT and the threshold Lipiodol uptake value shown in our study may enable intraprocedural assessment of TACE endpoints. This may prove beneficial in deciding when additional therapy is needed and to facilitate a treatment strategy following TACE, such as early repeat intervention or treatment using a different modality (i.e. ablation or $\mathrm{Y} 90$ radioembolization). 
There are a few limitations in this study. Because of the retrospective nature and strict inclusion/exclusion criteria of this study to rigorously detect a baseline Lipiodol threshold uptake value, the size of study population was limited and the follow-up period was relatively short. Although the baseline Lipiodol uptake was defined as HUs on the CT within one week after TACE, the HUs might vary by the timing of CT even during one week; e.g., the HUs on the 1st and the 7th day after TACE might be different. The concentration of the chemotherapeutic agents and the volume of Lipiodol injected was different by the case, which might potentially affect the treatment outcome, although the purpose of this study was simply to evaluate the association of the baseline Lipiodol uptake, which was obtained as the results of our practice, with the tumor recurrence. In addition, we excluded lesions treated with additional embolic materials. As mentioned above, we do not use the embolic particles routinely because we did not find a survival advantage with the use of particulate embolization (data not shown). This practice would be different from so-called conventional TACE, in which chemo-Lipiodol injection is usually followed by injection of additional embolic particles such as gelatin sponge. A recent randomized trial by Shi et al. showed no difference in survival outcomes between TACE with and without additional embolic materials following chemo-Lipiodol injection. ${ }^{22} \mathrm{On}$ the contrary, a large cohort study by Takayasu et al. showed that TACE with gelatin sponge embolization was associated with a higher survival rate than was a therapy without the particulate embolization. ${ }^{23}$ Thus, the significance of the particulate embolization in TACE seems still controversial and needs further investigation. Nevertheless, in the present study, the cohort of Lipiodol only TACE without embolic particles allowed us to analyze significance of Lipiodol uptake without being influenced by the embolic effect of other materials. This practice and our findings, however, may not extrapolate to a population treated with TACE followed by additional particulate embolization.

\section{Conclusions}

In conclusion, we demonstrated that Lipiodol uptake at baseline and Lipiodol washout rates at follow-up independently predicted tumor response after TACE for HCC. The critical threshold for baseline Lipiodol uptake predicting tumor recurrence was 270.2 HU. The association between baseline Lipiodol uptake and tumor response may prove useful for determining subsequent treatment strategies after Lipiodol TACE.

\section{Acknowledgments}

Khashayar Farsad received a grant from Guerbet for this study.

\section{References}

1. Bruix J, Sherman M. Management of hepatocellular carcinoma: an update. Hepatology 2011; 53: 1020-22. doi: 10.1002/hep.24199

2. Llovet JM, Bruix J. Systematic review of randomized trials for unresectable hepatocellular carcinoma: Chemoembolization improves survival. Hepatology 2003; 37: 429-42. doi: 10.1053/jhep.2003.50047

3. Idee JM, Guiu B. Use of Lipiodol as a drug-delivery system for transcatheter arterial chemoembolization of hepatocellular carcinoma: a review. Crit Rev Oncol Hematol 2013; 88: 530-49. doi: 10.1016/j.critrevonc.2013.07.003

4. Nakakuma K, Tashiro S, Hiraoka T, Uemura K, Konno T, Miyauchi Y, et al. Studies on anticancer treatment with an oily anticancer drug injected into the ligated feeding hepatic artery for liver cancer. Cancer 1983; 52: 2193200

5. Iwai K, Maeda $\mathrm{H}$, Konno T. Use of oily contrast medium for selective drug targeting to tumor: enhanced therapeutic effect and X-ray image. Cancer Res 1984; 44: 2115-21.

6. Ohishi $H$, Uchida $H$, Yoshimura $H$, Ohue $S$, Ueda J, Katsuragi $M$, et al. Hepatocellular carcinoma detected by iodized oil. Use of anticancer agents. Radiology 1985; 154: 25-9. doi: 10.1148/radiology.154.1.2981114

7. Kan Z, Madoff DC. Liver anatomy: microcirculation of the liver. Semin Intervent Radiol 2008; 25: 77-85. doi: 10.1055/s-2008-1076685

8. Jinno K, Moriwaki S, Tanada M, Wada T, Mandai K, Okada Y Clinicopathological study on combination therapy consisting of arterial infusion of Lipiodol-dissolved SMANCS and transcatheter arterial embolization for hepatocellular carcinoma. Cancer Chemother Pharmacol 1992; 31(Suppl): S7-12.

9. Choi BI, Kim HC, Han JK, Park JH, Kim YI, Kim, ST, et al. Therapeutic effect of transcatheter oily chemoembolization therapy for encapsulated nodular hepatocellular carcinoma: CT and pathologic findings. Radiology 1992; 182: 709-13. doi: 10.1148/radiology.182.3.1311116

10. Takayasu K, Arii S, Matsuo N, Ryu M, Takasaki K, Sato M, et al. Comparison of CT findings with resected specimens after chemoembolization with iodized oil for hepatocellular carcinoma. AJR Am J Roentgenol 2000; 175: 699-704. doi: 10.2214/ajr.175.3.1750699

11. Kwan SW, Fidelman N, Ma E, Kerlan RK, Jr., Yao FY. Imaging predictors of the response to transarterial chemoembolization in patients with hepatocellular carcinoma: a radiological-pathological correlation. Liver Transpl 2012; 18: 727-36. doi: 10.1002/It.23413

12. Imaeda $T$, Yamawaki $Y$, Seki $M$, Goto $H$, linuma $G$, Kanematsu $M$, et al. Lipiodol retention and massive necrosis after Lipiodol-chemoembolization of hepatocellular carcinoma: correlation between computed tomography and histopathology. Cardiovasc Intervent Radiol 1993; 16: 209-13.

13. Takayasu K, Muramatsu Y, Maeda T, Iwata R, Furukawa H, Muramatsu Y, et al. Targeted transarterial oily chemoembolization for small foci of hepatocellular carcinoma using a unified helical CT and angiography system: analysis of factors affecting local recurrence and survival rates. AJR Am J Roentgenol 2001; 176: 681-8. doi: 10.2214/ajr.176.3.1760681

14. Rou WS, Lee BS, Moon HS, Lee ES, Kim SH, Lee HY. Risk factors and therapeutic results of early local recurrence after transcatheter arterial chemoembolization. World J Gastroenterol 2014; 20: 6995-7004. doi: 10.3748/ wjg.v20.i22.6995 
15. Kinugasa $\mathrm{H}$, Nouso $\mathrm{K}$, Takeuchi $\mathrm{Y}$, Yasunaka T, Onishi $\mathrm{H}$, Nakamura $\mathrm{S}$, et al. Risk factors for recurrence after transarterial chemoembolization for early-stage hepatocellular carcinoma. J Gastroenterol 2012; 47: 421-6. doi: 10.1007/s00535-011-0492-9

16. European Association for the Study of The Liver, European Organisation for Rsearch and Treatment of Cancer. EASL-EORTC clinical practice guidelines: management of hepatocellular carcinoma. J Hepatol 2012; 56: 908-43. doi: 10.1016/j.jhep.2011.12.001

17. The Japan Society of Hepatology. Clinical Practice Guidelines for Hepatocellular Carcinoma 2013. [citated 2015 Sep 04]. Avalable at: http:// www.jsh.or.jp/English/guidelines_en/Guidelines_for_hepatocellular_carcinoma_2013.

18. Becker G. Creating comparability among reliability coefficients: the case of Cronbach alpha and Cohen kappa. Psychol Rep 2000; 87: 1171-82. doi: 10.2466/pr0.2000.87.3f.1171

19. Harvill LM. Standard error of measurement. Educational measurement: Issues and practice 1991; 10: 33-41. doi: 10.1111/j.1745-3992.1991. tb00195.x

20. Herber S, Biesterfeld S, Franz U, Schneider J, Thies J, Schuchmann M, et al. Correlation of multislice CT and histomorphology in HCC following TACE: predictors of outcome. Cardiovasc Intervent Radiol 2008; 31: 768-77. doi: $10.1007 /$ s00270-007-9270-8

21. Wang Z, Lin M, Lesage D, Chen R, Chapiro J, Gu T, et al. Three-dimensional evaluation of Lipiodol retention in HCC after chemoembolization: a quantitative comparison between CBCT and MDCT. Acad Radiol 2014; 21: 393-9. doi: 10.1016/j.acra.2013.11.006

22. Shi M, Lu LG, Fang WQ, Guo RP, Chen MS, Li Y, et al. Roles played by chemolipiodolization and embolization in chemoembolization for hepatocellular carcinoma: single-blind, randomized trial. J Nat/ Cancer Inst 2013; 105: 5968. doi: 10.1093/jnci/djs464

23. Takayasu K, Arii S, Ikai I, Kudo M, Matsuyama Y, Kojiro M, et al. Overal survival after transarterial lipiodol infusion chemotherapy with or without embolization for unresectable hepatocellular carcinoma: propensity score analysis. AJR Am J Roentgenol 2010; 194: 830-7. doi: 10.2214/AJR.09.3308 